\title{
Hypericum perforatum Extrakt LI 160: Ein Praxiserfahrungsbericht (PEB) über die Anwendung von Johanniskraut bei depressiven und körperlichen Beschwerden
}

\author{
Serge Brand, Johannes Beck, Edith Holsboer-Trachsler \\ Universitäre Psychiatrische Kliniken UPK Basel, Schweiz
}

D ie Wirksamkeit von Johanniskrautextrakt LI 160 (MeOH 80\%; Hypericum perforatum; St. John's wort) zur Verminderung depressiver Symptome wurde früher wiederholt und in mehreren doppelblind und placebokontrolliert durchgeführten Untersuchungen gezeigt $[1,2,3]$. Weitere Studien mit einem ähnlichen methanolischen Extrakt belegen die Wirksamkeit von Johanniskraut [4, 5]. So zeigten KASPER et al. die Überlegenheit von Hypericum perforatum in täglichen Dosierungen von $600 \mathrm{mg}$ und $1200 \mathrm{mg}$ gegenüber Placebo sowohl für die Akutbehandlung wie auch als Erhaltungstherapie nach Remission [5] von milden oder moderaten depressiven Störungen. FAVA et al. referierten die Überlegenheit von Hypericum perforatum (LI 160; $900 \mathrm{mg} / \mathrm{d}$ ) gegenüber Fluoxetin und Placebo nach 12-wöchiger Behandlung von depressiven Störungen [6]. Weiter konnten FindLING et al. [7] in einer prospektiven Studie zeigen, dass Hypericum perforatum auch bei juveniler Depression wirkte. Diese Ergebnisse wurden in einer Anwendungsbeobachtung mit dem Johanniskrautextrakt LI 160 mit 10'627 Patienten bestätigt [8]. Mittlerweile liegen nicht weniger als fünf Metaanalysen und Zusammenfassungen zu Hypericum perforatum vor [9-13], welche die Wirksamkeit und Verträglichkeit von Johanniskraut zur Behandlung von leichten [9, 10, 12-13], moderaten [9, 10, 12-13], bis mittelschweren [9, 11] depressiven Störungen belegen. In Kontrast hierzu hielten LiNDE et al. [14]
Hintergrund: Placebokontrollierte Untersuchungen zeigten eine gute Wirksamkeit des Johanniskrautextraktes LI 160 bei der Behandlung von Patienten mit Depressionen wie auch mit somatoformen Störungen. Fragestellung: Dieser Praxiserfahrungsbericht soll die Anwendung von Hypericum bei Patienten mit Depressionen und körperlichen Symptomen prüfen, die Applikation in verschiedenen Altersklassen darlegen und die Bedeutung der Begleitmedikation darstellen. Methode: Insgesamt wurden 725 Patienten mit Johanniskrautextrakt LI 160 (2x 450 mg pro Tag) behandelt, und Daten von einem standardisierten Fragebogen wurden bei behandelnden Ärzten zu Beginn und am Abschluss einer vier- bis sechswöchigen Behandlung erhoben. Ergebnisse: Hypericum bewirkte eine von Alter und Geschlecht unabhängige deutliche Verringerung der depressiven Symptomatik und der somatischen Symptome. Patienten mit schwereren depressiven und körperlichen Symptomen erhielten häufiger eine Begleitmedikation. Die Zufriedenheit mit der Therapie mit Hypericum perforatum lag bei 88\% für die Wirksamkeit und bei 98\% für die Verträglichkeit. Schlussfolgerungen: Die Zufriedenheit mit Hypericum (LI 160) war bei Patienten mit milden und moderaten depressiven und somatoformen Symptomen alters- und geschlechtsunabhängig gut. Depressive und körperliche Symptome treten im klinischen Alltag häufig überlappend auf. Bei schwerer Symptomatik werden häufiger synthetische Antidepressiva eingesetzt.

Schlüsselwörter: Hypericum perforatum, depressive Symptome, körperliche Begleitsymptome, LI 160, somatoforme Symptome, Praxiserfahrungsbericht

\section{Hypericum perforatum extract LI 160: An Observational Study on Treatment of Depressive and Somatic Symptoms with St. John's Wort}

Background: Placebo-controlled trials indicate a high level of efficacy with the use of Hypericum perforatum (Li 160) in the treatment of patients with depressive and somatoform disorders. Aim of the study: To investigate the effects of hypericum in the treatment of both depressive and somatic symptoms for different ages of patients as well as the importance of concomitant medication. Methods: A total of 725 patients in 145 physician's practices who were being treated with the St. John's wort extract LI 160 (2x 450 mg per day) were given a standardized questionnaire and data were collected at the beginning and at the end of treatment with hypericum. Treatment lasted from four to six weeks. Results: Beneficial effects of hypericum on both depressive and somatoform symptoms were observed independently of age and gender. Patients with highly increased depressive and somatoform scores needed concomitant medication. Satisfaction with efficacy reached $88 \%$, and satisfaction with tolerability reached $98 \%$. Conclusions: Independently of age and gender, hypericum is well tolerated and regarded highly effective for both mild to moderate depressive and somatic symptoms. Patients suffering from more severe symptoms seem to need concomitant medication or a switch to synthetic antidepressive medication.

Keywords: Hypericum perforatum, depressive symptoms, LI 160, somatoform symptoms, observational study 
in ihrer Metaanalyse fest, dass die Evidenz zur Wirkung von Hypericum perforatum zur Behandlung von depressiven Störungen inkonsistent sei; dieses heterogene Bild sei wohl auf Unterschiede der angewendeten Methoden sowie der Produkte mit sehr heterogener pharmazeutischer Qualität zurückzuführen (vgl. auch [15]).

Depressive und somatoforme Störungen weisen im klinischen Bild Gemeinsamkeiten auf. So beziehen sich nicht weniger als sechs von 17 Items der im klinischen Alltag häufig verwendeten Hamilton-Rating Scale zur Fremdeinschätzung depressiver Störungen auf somatische Symptome (vgl. [16]). Entsprechend konnte die günstige Wirkung von Hypericum auf somatoforme Störungen belegt werden [9, 17, 18].

Für die Wirksamkeit von Johanniskraut wurden mehrere Mechanismen und unterstützende Wirkmechanismen beschrieben [19]. Als möglicher Wirkmechanismus wird eine verstärkte Expression von p-Glycoproteinen durch Hypericum angenommen (vgl. [20]). P-Glycoprotein ist eine Pumpe in Zellen der Blut-Hirn-Schranke, die Giftstoffe, Medikamente, aber auch das Stresshormon Cortisol aus dem ZNS heraustransportiert. So könnte Hypericum die Menge an intracerebralen Corticosteroiden verringern, was eine beruhigende Wirkung auf die bei depressiven Patienten überaktive Stresshormonachse ausübt.

Zusammenfassend liegt eine Vielzahl an Befunden vor, die eine Wirksamkeit von Hypericum (LI 160) auf depressive Symptome bei Erwachsenen, Kindern und Adoleszenten sowie auch somatoforme Störungen bei Erwachsenen zeigen; gleichzeitig wurden gute Verträglichkeit und sehr geringe unerwünschte Arzneimittelwirkungen (UAW's) beschrieben (vgl. [9-13]).

Der vorliegende Praxiserfahrungsbericht soll Daten über die Anwendung von Hypericum bei gleichzeitig auftretenden depressiven und körperlichen Symptomen erheben und untersuchen, ob die therapeutische Wirkung altersabhängig ist. Zudem wurden Daten zu Begleitmedikation, unerwünschten Arzneimittelwirkungen
(UAW), Zufriedenheit mit Wirkung und Verträglichkeit sowie auch zu Studienabbruch erhoben und ausgewertet.

\section{Methoden}

\section{Durchführung}

Die Datenerhebung fand zwischen Oktober 2006 und Juni 2007 in verschiedenen Kantonen der deutschen, französischen und italienischen Schweiz statt. Die Ethikkommissionen der entsprechenden Kantone wurden für die Durchführung des Praxiserfahrungsberichtes angefragt und entsprechend informiert und dokumentiert. Die Notifizierung eines Praxiserfahrungsberichts zuhanden der Arzneimittelbehörde war nicht notwendig. Niedergelassene Ärzte behandelten Patienten jeden Alters, welche über depressive Symptome klagten; die teilnehmenden Ärzte waren in der Wahl der Medikation und in der entsprechenden Dosierung frei. Der Einsatz der Medikamente erfolgte nach den in der Fachinformation beschriebenen Empfehlungen im Rahmen der täglichen Praxis. Es fand eine Visite $\mathrm{zu}$ Beginn und am Ende der in der Fachinformation empfohlenen vier- bis sechswöchigen Behandlung statt.

Insgesamt wurden die Daten von 725 Patientinnen und Patienten durch 145 Ärztinnen und Ärzte erhoben: 92 Allgemeinpraktiker, 33 Internisten, 13 Psychiater, 2 Chirurgen, 2 Rheumatologen, 2 Gynäkologen, 1 Gastroenterologe.

\section{Diagnose und erhobene Daten}

Zur Diagnose einer depressiven und körperlichen Symptomatik wurden die Kriterien nach DSM-IV [21] zugezogen (vgl. Tabelle 3). Die körperliche Symptomatik wurde in Anlehnung an die somatischen Items der Hamilton Anxiety Scale (HAMAsom; vgl. [17, 18] vorgeschlagen. $\mathrm{Zu}$ Beginn und nach Abschluss der in der Fachinformation empfohlenen vier- bis sechswöchigen Therapie wurden die Daten zu depressiven und körperlichen Symptomen mittels fünfstufigen Likert-Skalen mit den Ankerpunkten 0 (= nicht vorhanden), 1 (= gering), 2 (= mässig), 3 (= stark) und 4 (= sehr stark) ermittelt.
Erhöhte Mittelwerte implizierten ausgeprägtere depressive oder körperliche Symptome. Zusätzlich wurden neben den demographischen Daten (Tabelle 1) die Begleitmedikation (Antwortmöglichkeiten: ja-nein), die subjektive Zufriedenheit ("mit der Wirksamkeit”: ja-nein; "mit der Verträglichkeit”: ja-nein), unerwünschte Arzneimittelwirkungen (UAW; ja-nein; Art, Dauer, Ausgang), ein möglicher vorzeitiger Behandlungsabbruch bzw. eine vorzeitige Behandlungsbeendigung (ja-nein; Grund) sowie die Option erfasst, die Behandlung mit dem Präparat weiterzuführen.

Um Aussagen über die Veränderungen der depressiven und körperlichen Symptomatik in Alterskategorien zu machen, wurden Altersklassen wie in Tabelle 2 angegeben verwendet.

\section{Medikation}

Filmtabletten mit $450 \mathrm{mg}$ Johanniskrautextrakt LI 160 (Jarsin $\left.{ }^{\circledR} 450\right)$ (Hyperici herbae extractum); DrogeExtrakt-Verhältnis 3-6:1; Auszugsmittel $80 \%$ Vol. Methanol, quantifiziert auf $0.54-1.26 \mathrm{mg}$ Gesamthypericin. Wenn nicht anders verschrieben, werden zwei Filmtabletten täglich eingenommen.

\section{Statistische Methoden}

Demographische Unterschiede zwischen männlichen und weiblichen $\mathrm{Pa}$ tienten wurden mittels t-Tests ermittelt. Mittelwertsunterschiede der depressiven und körperlichen Symptomatik zu Beginn und nach Abschluss der Behandlung (Prä-Post-Vergleiche) wurden mittels t-Tests für verbundene Stichproben errechnet. Effektstärken wurden nach den gepoolten Standardabweichungen berechnet, wobei nach BorTz und DörIng [22] die Effektstärken wie folgt bewertet wurden: $d>0.3$ $=$ kleiner Effekt, $\mathrm{d}>0.6=$ mittlerer Effekt, $d>0.9=$ grosser Effekt. ANOVAs wurden durchgeführt, um Prä-PostVergleiche in Abhängigkeit vom Vorliegen einer Begleitmedikation oder in Abhängigkeit von Altersklassen zu berechnen. Häufigkeitsunterschiede wurden mittels Chi-Quadrat-Berechnungen durchgeführt.

MANCOVAs (ANOVAs für Messwiederholungen und mit Kovariaten) wur- 
Tab. 1. Demographische Daten vor Beginn des Praxiserfahrungsberichtes $(n=725)$

\begin{tabular}{|c|c|c|c|}
\hline & Patienten & & Statistik \\
\hline Dimensionen & Weibliche (n = 496) & Männliche ( $n=229$ ) & t-Tests / Chi-Quadrat-Test \\
\hline Alter [Jahre M (SD)] & $48.25(16.56)$ & $47.22(15.63)$ & $t(723)=-0.71, p=0.480$ \\
\hline Grösse [cm M (SD)] & $164.38 \quad(6.58)$ & $174.70 \quad(7.86)$ & $\mathrm{t}(723)=18.32, \mathrm{p}<0.001$ \\
\hline Gewicht [kg M (SD)] & $65.38(13.72)$ & $77.55(11.80)$ & $\mathrm{t}(723)=11.50, p<0.001$ \\
\hline BMI [M (SD)] & $24.26 \quad(5.27)$ & $25.34 \quad(3.30)$ & $t(723)=2.81, p=0.001$ \\
\hline
\end{tabular}

Tab. 2. Altersklassen und Häufigkeitsverteilung $(n=725)$

\begin{tabular}{|c|c|c|}
\hline Altersklassen in Jahren & Anzahl Befragte & Alterskategorien \\
\hline $13-20$ & 19 & $\begin{array}{l}\text { Adoleszenz } \\
\text { und frühes Erwachsenenalter }\end{array}$ \\
\hline $21-40$ & 229 & Frühes Erwachsenenalter \\
\hline $41-60$ & 316 & Mittleres Erwachsenenalter \\
\hline $61-80$ & 142 & Spätes Erwachsenenalter \\
\hline $81-94$ & 19 & Sehr spätes Erwachsenenalter \\
\hline
\end{tabular}

Tab. 3. Depressive und körperliche Symptome zu Beginn und nach der Behandlung $(n=725)$

\begin{tabular}{|c|c|c|c|}
\hline Depressive Symptomatik & Beginn & Ende & p-Wert \\
\hline Gedrückte Stimmung & $3.16(0.84)$ & $2.08(1.09)$ & $\mathrm{p}<0.001$ \\
\hline Stimmungslabilität & $2.98(0.95)$ & $1.98(0.81)$ & $\mathrm{p}<0.001$ \\
\hline Antriebsmangel & $3.12(1.00)$ & $2.02(0.86)$ & $\mathrm{p}<0.001$ \\
\hline Unausgeglichenheit & $2.92(1.00)$ & $1.89(0.84)$ & $\mathrm{p}<0.001$ \\
\hline Innere Unruhe/Ängstlichkeit & $3.19(0.99)$ & $2.01(0.87)$ & $\mathrm{p}<0.001$ \\
\hline Reizbarkeit/Nervosität & $3.05(1.03)$ & $1.94(0.82)$ & $\mathrm{p}<0.001$ \\
\hline Einschlafstörungen & $2.83(1.09)$ & $1.78(0.82)$ & $\mathrm{p}<0.001$ \\
\hline Durchschlafstörungen & $2.78(1.09)$ & $1.75(1.13)$ & $\mathrm{p}<0.001$ \\
\hline Erschöpfung & $2.95(1.15)$ & $1.83(0.87)$ & $\mathrm{p}<0.001$ \\
\hline $\begin{array}{l}\text { Mittelwert } \\
\text { aller depressiven Symptome }\end{array}$ & $2.99(0.68)$ & $1.92(0.64)$ & $\mathrm{p}<0.001$ \\
\hline Körperliche Symptomatik & Beginn & Ende & p-Wert \\
\hline Muskuläre Symptome & $2.07(1.05)$ & $1.47(0.70)$ & $\mathrm{p}<0.001$ \\
\hline Sensorische Symptome & $1.97(1.00)$ & $1.42(0.66)$ & $\mathrm{p}<0.001$ \\
\hline Kardiovaskuläre Symptome & $2.09(1.05)$ & $1.41(0.66)$ & $\mathrm{p}<0.001$ \\
\hline Respiratorische Symptome & $1.99(1.00)$ & $1.4(1.03)$ & $\mathrm{p}<0.001$ \\
\hline Gastrointestinale Symptome & $2.21(1.05)$ & $1.48(0.72)$ & $\mathrm{p}<0.001$ \\
\hline Urogenitale Symptome & $1.69(0.94)$ & $1.32(0.62)$ & $\mathrm{p}<0.001$ \\
\hline Neurovegetative Symptome & $2.18(1.05)$ & $1.48(0.69)$ & $\mathrm{p}<0.001$ \\
\hline $\begin{array}{l}\text { Mittelwert } \\
\text { aller körperlichen Symptome }\end{array}$ & $2.03(0.72)$ & $1.43(0.48)$ & $p<0.001$ \\
\hline
\end{tabular}

den durchgeführt, um Prä-Post-Vergleiche in Abhängigkeit von Altersklassen (vgl. Tabelle 2) zu berechnen;

\section{Ergebnisse}

Die demographischen Daten sind in Tabelle 1 zusammenfassend dargestellt. Patienten wiesen gegenüber Patientinnen eine grössere Körpergrösse, ein höheres Gewicht sowie einen höheren Body Mass Index auf. Es lagen komplette Datensätze für alle Patienten vor, also auch für jene mit frühzeitiger Beendigung oder mit einem Abbruch der Studie. Alle Patienten wurden mit dem HypericumExtrakt Jarsin ${ }^{\circledR} 450$ behandelt. Die verschriebene Tagesdosis variierte zwischen 1 und 3 Filmtabletten. Es wurde kein systematischer Zusammenhang zwischen Tagesdosis und Geschlecht $\left(\chi^{2}(3)=0.63, p=.73\right)$, Altersklasse $\left(\chi^{2}(8)=12.97, p=0.12\right)$ oder Begleitmedikation $\left(\chi^{2}(2)=1.29, \mathrm{p}=0.53\right)$ beobachtet.

\section{Depressive und körperliche Symptome zu Beginn und nach Beendigung der Behandlung}

In Tabelle 3 sind die deskriptiven und inferentialstatistischen Angaben der depressiven und körperlichen Symptome angegeben. Zusammengefasst kann festgehalten werden, dass sich die depressive und körperliche Symptomatik im Laufe der vier- bis sechswöchigen Behandlung signifikant verbesserte (Abbildung 1). Die Effektstärken (depressive Symptomatik: $d=1.63$; körperliche Symptomatik: $d=1.00$ ) sind sehr hoch. Eine spontane Verbesserung der Symptomatik in dieser Grössenordnung ist nicht zu erwarten [23] und demzufolge sind die Mittelwertsveränderungen mit grosser Wahrscheinlichkeit auf die Behandlung zurückzuführen. 


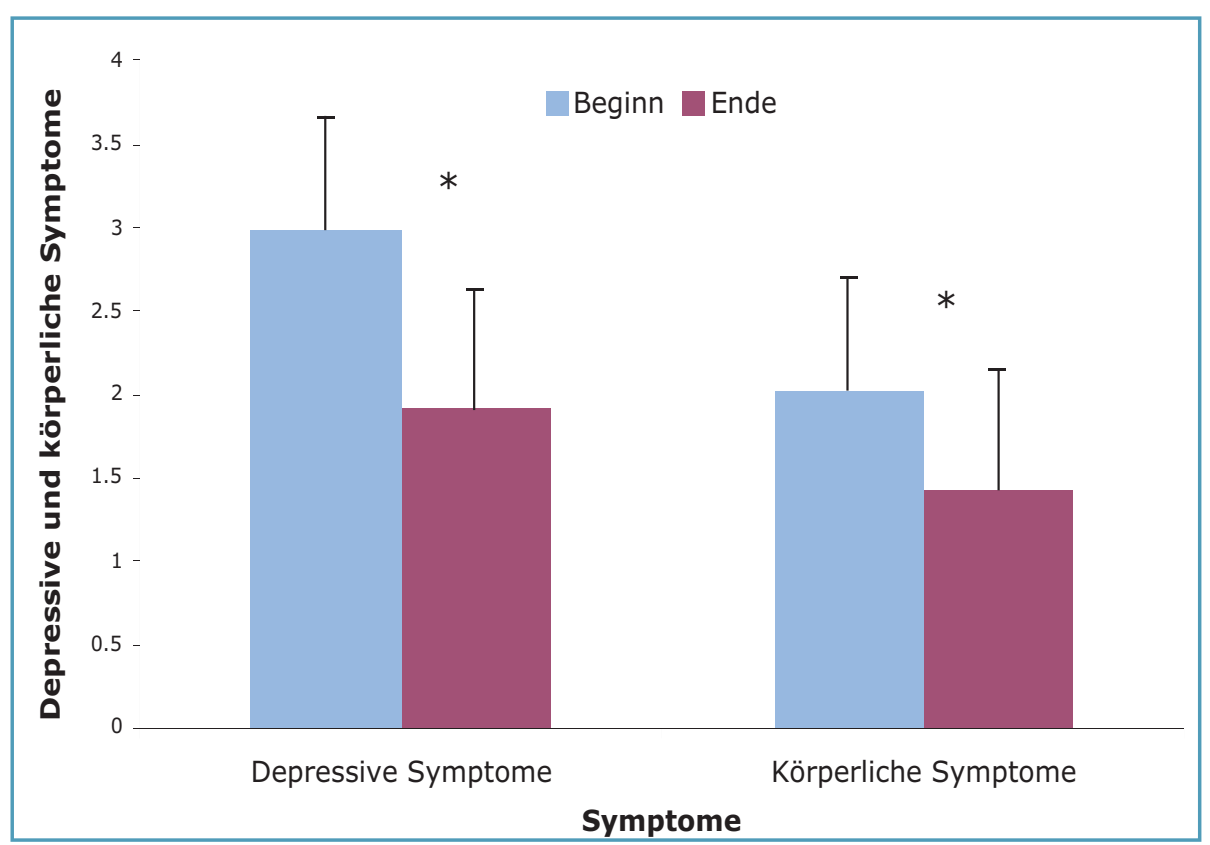

Abb. 1. Depressive und körperliche Symptome zu Beginn und am Ende der Behandlung, Mittelwerte und Standardabweichungen; ${ }^{*}=p<0.001$

\section{Begleitmedikation}

Von den 725 behandelten Patienten nahmen $37.1 \%$ eine Begleitmedikation ein, $57.7 \%$ nahmen keine Begleitmedikation. Bei $5.2 \%$ blieb die Frage unbeantwortet. Als Begleitmedikation galt die Einnahme von Sedativa, Antidepressiva und/oder Anxiolytika.

Wer eine Begleitmedikation hatte, wies zu Beginn (3.13 \pm 0.64) und am Ende der Behandlung (2.02 \pm 0.67) signifikant höhere Werte der depressiven Symptome auf als Befragte ohne Begleitmedikation (Beginn: $2.94 \pm$ 0.68; Ende: $1.85 \pm 0.60$ ): Der Faktor Prä-Post-Vergleich (Stufen: Beginn, Ende der Behandlung) war signifikant $(\mathrm{F}(1,685)=1759.08, \mathrm{p}<0.001)$, der Faktor Begleitmedikation (Stufen: Begleitmedikation ja-nein) war ebenfalls signifikant $(\mathrm{F}(1,685)=17.50, \mathrm{p}<0.001)$; die Interaktion Prä-Post-Vergleich $\mathrm{x}$ Begleitmedikation war nicht signifikant $(\mathrm{F}(1,685)=0.00, \mathrm{p}=0.99)$.

Das identische Ergebnismuster zeigte sich ebenfalls bezüglich der körperlichen Symptome: Wer eine Begleitmedikation hatte, wies zu Beginn (2.18 \pm 0.69) und am Ende der Behandlung (1.52 \pm 0.49$)$ signifikant höhere Werte der körperlichen Begleitsymptome auf als Befragte ohne Begleitmedikation (Beginn: $1.95 \pm 0.75$; Ende: $1.36 \pm 0.46$ ) . hängigkeit der Altersklassen (Abbildung 2).

\section{Altersklassen und körperliche Symptome}

Es wurde eine MANCOVA wie oben referiert berechnet: Der Faktor Altersklasse war signifikant $(\mathrm{F}(4,681)=$ 17.07, $\mathrm{p}<0.001$ ); der Faktor Messwiederholung war signifikant $(\mathrm{F}(1,681)=$ 67.41, p < 0.001); der Faktor Begleitmedikation war signifikant $(\mathrm{F}(1,681)=$ 4.34, $\mathrm{p}<0.001)$; die Interaktionen waren nicht signifikant (alle F's < 1.5). Somit veränderten sich die Werte der körperlichen Beschwerden in Abhängigkeit vom Messzeitpunkt (höhere Werte zu Beginn der Behandlung), in Abhängigkeit der Begleitmedikation (höhere Werte bei Begleitmedikation), und in Abhängigkeit der Altersklasse (höhere Werte in älteren Altersklassen: vgl. Abbildung 3).

\section{Unerwünschte}

\section{Arzneimittelwirkungen (UAW)}

Von den 725 Erhebungen lagen bei 7\% keine Angaben vor, $90.7 \%$ gaben keine, und $2.3 \%$ gaben unerwünschte Arzneimittelwirkungen an. Diese Nebenwirkungen betrafen vor allem gastrointestinale Beschwerden (Blähungen, Völlegefühl, Bauchschmerzen, Durchfall, aber auch Verstopfung (40\% aller UAW’s), Kopfschmerzen/Migräne (20\%), Übelkeit (20\%), Müdigkeit (10\%) und allergische Reaktion (10\%). In der Regel klangen die UAW's nach ein bis sieben Tagen ab. Weiter wurden keine schwerwiegenden oder unbekannten UAW's beobachtet. Die hier beobachtete Nebenwirkungsrate entspricht der Rate von früheren grösseren Anwendungsbeobachtungen [8].

\section{Zufriedenheit mit der Wirksamkeit und der Verträglichkeit}

$88.3 \%$ der Patienten waren mit der Wirksamkeit der Hypericum-Medikation zufrieden; $10.3 \%$ waren nicht zufrieden. Bei 1.4\% fehlten die Angaben. $96.7 \%$ der Patienten waren mit der Verträglichkeit zufrieden; 1.9\% waren unzufrieden.

Die Zufriedenheit mit der Wirksamkeit war weder geschlechts- noch altersabhängig. Die Patienten ohne Begleitmedikation waren mit der The- 


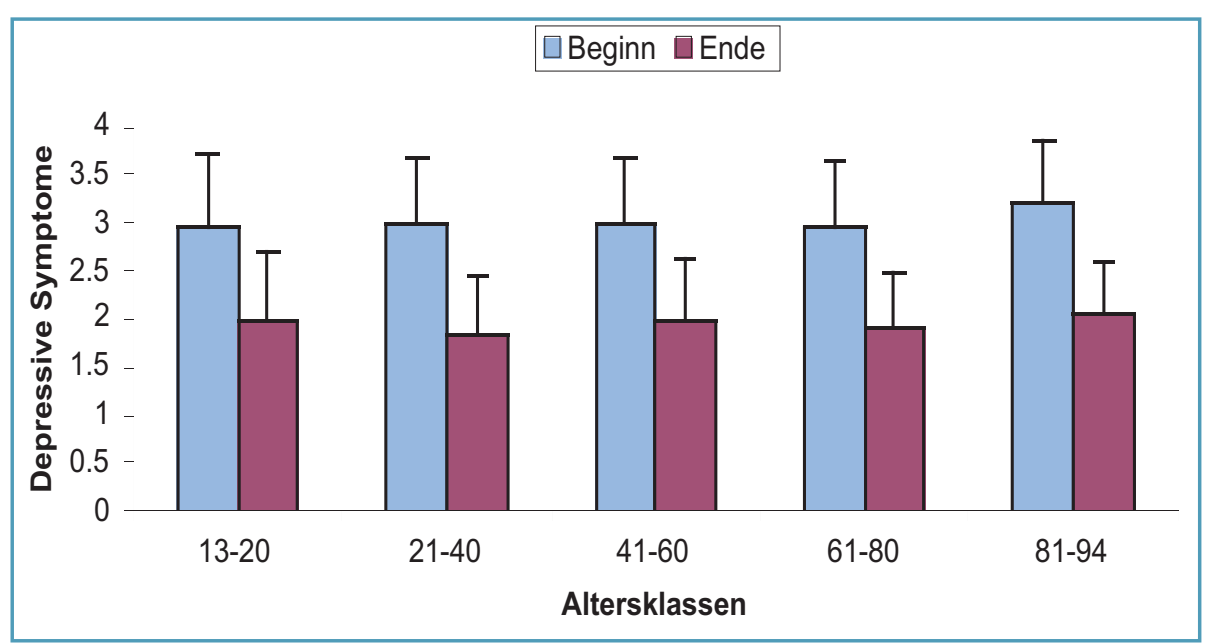

Abb. 2. Depressive Symptome in den fünf Altersklassen zu Beginn und am Ende der Behandlung, Mittelwerte und Standardabweichungen.

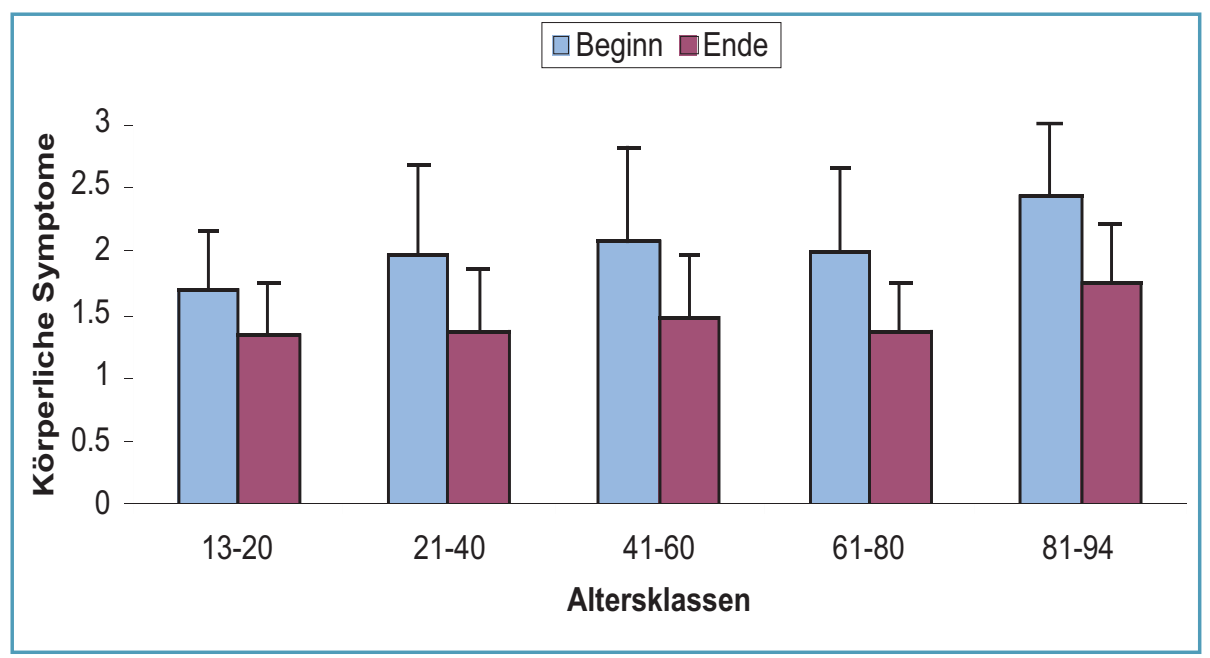

Abb. 3. Körperliche Symptome in den fünf Altersklassen zu Beginn und am Ende der Behandlung, Mittelwerte und Standardabweichungen.

rapie signifikant zufriedener $\left(\chi^{2}(1)=\right.$ $6.66, \mathrm{p}=0.01)$.

\section{Vorzeitige Therapiebeendigung}

Von den 725 Patienten brachen 9.1\%, also 66 Patienten, die Behandlung vor Abschluss der regulären Therapiezeit ab. Vier gaben körperliche Symptome als Nebenwirkung an (Allergie im Nacken, Blähungen und Bauchschmerzen, Durchfall, Migräne). Bei zweiundfünfzig Patienten gab es keine Verbesserung, bzw. wurde eine Verschlechterung der Symptomatik empfunden; bei 10 Erhebungen wurden keine Angaben gemacht.

Dreiundzwanzig Patienten (3.2\%) beendeten die Therapie vorzeitig auf- grund der vollständig abgeklungenen Symptome.

\section{Weiterführende Behandlung mit Johanniskrautextrakt LI $\mathbf{1 6 0}$}

Bei 571 Patienten (79\%) wurde die Behandlung mit Johanniskrautextrakt LI 160 weitergeführt; von 26 (3.6\%) fehlten die Angaben; bei 128 Patienten $(17.7 \%)$ wurde die Behandlung mit Johanniskrautextrakt LI 160 nicht fortgeführt; von diesen haben 23 die erfolgreiche Therapie vorzeitig beendet; 66 brachen die Therapie vorzeitig ab, sodass bei 72 Patienten (9.9\%) ohne weitere Angaben von Gründen die Behandlung mit Johanniskrautextrakt LI 160 nicht weitergeführt wurde.

\section{Diskussion}

Der vorliegende Praxiserfahrungsbericht zeigte, dass eine vier- bis sechswöchige Behandlung mit Hypericum perforatum (Jarsin ${ }^{\circledR} 450$ Filmtabletten; LI 160) eine gute Wirksamkeit bei leichten bis mittelschweren depressiven Symptomen aufwies. Die depressionsverbessernde Wirkung konnte dabei altersunabhängig sowohl bei Präadoleszenten (vgl. [7]) wie auch bei Hochbetagten beobachtet werden. Demnach stimmen diese Resultate mit vorangegangenen Befunden überein, welche die günstige Wirkung von $\mathrm{Hy}$ pericum zur Behandlung von leichten bis moderaten depressiven Symptomen belegten [9, 10, 12-13]. Die vorliegenden Resultate stehen in Kontrast zu Befunden, welche die Wirkung von Hypericum (LI 160) in Frage stellen [14, 15].

Weiter konnte gezeigt werden, dass erhöhte depressive Symptome im Sinne einer mittelschweren Depression in der Praxis nicht mit einer HypericumMonotherapie behandelt wurden, sondern therapiebegleitend mit synthetischen Pharmaka. Insofern stehen vorliegende Ergebnisse eher in Kontrast zu Angaben, wonach Hypericum auch bei mittelschweren depressiven Symptomen indiziert sei $[9,11]$.

Ferner wiesen die Auswertungen darauf hin, dass keine systematischen Alters- und Geschlechtseffekte zu beobachten waren, ob und mit welcher Dosierung eine Mono- oder eine kombinierte Pharmakotherapie durchgeführt wurde; folglich kann davon ausgegangen werden, dass die vorliegende Ausprägung der Symptomatik bei der Gabe und Art der Medikation wohl ausschlaggebend war.

Ebenfalls konnte eine günstige Wirkung des Präparates bei körperlichen Symptomen beobachtet werden, was aufgrund vorangegangener Studien $[17,18] \mathrm{zu}$ erwarten war. Der Praxiserfahrungsbericht wies allerdings auch auf eine hohe Überlappung zwischen depressiven und körperlichen Symptomen hin: Diese Überlappung wird im Praxisalltag sehr häufig beobachtet und stellt oft eine differentialdiagnostische Herausforderung dar Konsequenterweise sollte beim Wunsch nach wiederholter diagnostischer $\mathrm{Ab}$ 
klärung bei aktuell und anamnestisch unauffälligen organischen Befunden an somatische Symptome einer Depression oder an eine somatoforme Störung gedacht werden; eine gezielte Exploration depressiver Symptome sollte erfolgen.

Die hohe Verträglichkeitsrate sowie die geringen und passageren Nebenwirkungen bestätigen die in der Literatur referierten Angaben (vgl. [1; 4-7, 9]): die Inzidenz von UAW's liegt zwischen 0 und $6 \%$ [9]; entsprechend kann die vorliegende Inzidenz von $2.3 \%$ als gering bewertet werden.

Vorzeitige Therapieabbrüche wurden dann beobachtet, wenn sich die Symptomatik nicht verbesserte, worauf eine Zusatzmedikation gegeben wurde oder ein Präparatewechsel erfolgte. Letzterer Fall war vor allem bei älteren Patienten mit initial erhöhten depressiven und körperlichen Symptomen zu sehen.

Zusammenfassend bestätigt dieser Praxiserfahrungsbericht die Ergebnisse früherer Studien, die Hypericum perforatum bei leichten und mittleren depressiven und körperlichen Symptomen als eine mögliche Therapieoption der ersten Wahl empfehlen, wohingegen bei Patienten mit schwerer depressiver Symptomatik der zusätzliche Einsatz synthetischer Pharmaka indiziert ist.

\section{Danksagung}

Vifor AG, Villars-sur-Glâne, hat den PEB durchgeführt (Dr. C. Terreaux). Die statistische Auswertung sowie die Analyse und Interpretation der Ergebnisse wurde im Auftrag des Sponsors durch die Abteilung Depressionsforschung, Schlafmedizin und Neurophysiologie der UPK Basel, (Dr. S. Brand, Dr. J. Beck, Prof. E. Holsboer-Trachsler) durchgeführt. Vifor AG hatte keinen Einfluss auf die Analysen der Resultate, auf deren Interpretation und auf die Einreichung des Manuskriptes.

\section{Literatur}

1. Hänsgen KD, Vesper J: Antidepressive Wirksamkeit eines hochdosierten HypericumExtraktes. Münch Med Wochenschr 1996; 138:35-39.

2. Vorbach EU, Arnoldt KH, Hübner WD: Efficacy and tolerability of St. John's wort extract LI 160 versus imipramine in patients with severe depressive episodes according to ICD-10. Pharmacopsychiatry 1997;30:81-85.

3. Wheatley D: LI 160, an extract of St. John's wort, versus amitriptyline in mildly to moderately depressed outpatients - a controlled 6week clinical trial. Pharmacopsychiatry 1997; 30:77-80

4. Lecrubier Y, Clerc G, Didi R, Kieser M: Efficacy of St. John's wort extract WS 5570 in major depression: a double-blind, placebocontrolled trial. Am J Psychiatry 2002;159: 1361-1366.

5. Kasper S, Anghelescu IG, Szegedi A, Dienel A, Kieser M: Placebo controlled continuation treatment with Hypericum extract WS 5570 after recovery from mild or moderate depressive episode. Wien Med Wochenschr 2007; 157:263-266

6. Fava M, Alpert J, Nierenberg AA, Mischoulon D, Otto MW, Zajecka J, Murck H, Rosenbaum JF: A double-blind, randomized trial of St John's wort, fluoxetine, and placebo in major depressive disorder. J Clin Psychopharmacol 2005;25:441-447.

7. Findling RL, McNamara NK, O'Riordan MA, Reed MD, Demeter CA, Branicky LA, Blumer JL: An open-label pilot study of St. John's wort in juvenile depression. J Am Acad Child Adolesc Psychiatry 2003;42:908-914.

8. Schulz V, Toelg M, Ploch M: Wirksamkeit und Verträglichkeit von Johanniskraut-Extrakt in hoher Einzeldosis. Z Phytother 2006;27:16-19.

9. Kraft K: Aktuelle Entwicklungen bei Johanniskrautextrakten: Neue Daten zu Wirksamkeit und Interaktionen. Wien Med Wochenschr 2007;157:284-287.

10. Clement $K$, Covertson CR, Johnson MJ, Dearing K: St. John's wort and the treatment of mild to moderate depression: a systematic review. Holist Nurs Pract 2006;20:197-203.

11. Röder C, Schaefer M, Leucht S: Meta-Analyse zu Wirksamkeit und Verträglichkeit der Behandlung der leichten und mittelschweren Depression bei Johanniskraut. Fortschr Neurol Psychiatr 2004;72:330-343

12. Schulz V: Clinical trials with Hypericum extracts in patients with depression - Results, comparisons, conclusions for therapy with antidepressant drugs. Phytomedicine 2002; 9:468-474.

13. Pilkington K, Boshnakova A, Richardson J: St John's wort for depression: Time for a different perspective? Complementary Ther Med 2006;14:268-281.

14. Linde K, Berner M, Egger M, Murlow C: St John's wort for depression. Br J Psychiatry 2005;186:99-107.

15. Hypericum Depression Trial Study Group:
Effect of Hypericum perforatum (St John's wort) in major depressive disorder. JAMA 2002; 287:1807-1814.

16. Farabaugh A, Mischoulon D, Fava , Wu SL, Mascarini A, Tossani E, Alpert JE: The relationship between early changes in the HAMD-17 anxiety/somatization factor items and treatment outcome among depressed outpatients. Int Clin Psychopharmacol 2005; 20:87-91

17. Müller T, Mannel M, Murck $H$, Rahlfs WW: Treatment of somatoform disorders with St. John's wort: a randomized, double-blind and placebo-controlled trial. Psychosom Med 2004;66:538-547

18. Volz HP, Murck H, Kasper S, Möller HJ: St John's wort extract (LI 160) in somatoform disorders: results of a placebo-controlled trial. Psychopharmacology 2002;164:294-300.

19. Butterweck V, Schmidt M: St. John's wort: role of active compounds for its mechanism of action and efficacy. Wien Med Wochenschr 2007;157:356-361.

20. Murck H: Atypical depression and related illnesses - neurobiological principles for their treatment with Hypericum extract. Wien Med Wochenschr 2002;152:398-403.

21. Sass H, Wittchen HU, Zaudig M, Houben I: Diagnostisches und Statistisches Manual zur Klassifikation Psychischer Störungen. Göttingen: Hogrefe, 2003.

22. Bortz J, Döring N: Forschungsmethoden und Evaluation für Human- und Sozialwissenschaftler. Springer, Heidelberg 2005.

23. Hegel MT, Oxman TE, Hull JG, Swain K, Swick $\mathrm{H}$. Watchful waiting for minor depression in primary care: remission rates and predictors of improvement. Gen Hosp Psychiatry 2006;28:205-212

\section{Korrespondenzadresse:}

Prof. Dr. med. Edith Holsboer-Trachsler Universitäre Psychiatrische Kliniken Basel Depressionsforschung, Schlafmedizin und Neurophysiologie

Wilhelm Kleinstrasse 27, CH-4025 Basel

Tel.: 0041 (0)613255097

Fax: 0041 (0)613255 513

Wissenschaftliches Sekretariat:

Christine.haselbach@upkbs.ch 